\title{
Tecnología para el mejoramiento educacional (TPEME)
}

\section{Technology for educational improvement (TPEME)}

\author{
Sophia Atencio ${ }^{l}$, Jonathan Cárcamo ${ }^{1}$, Raúl Fernández ${ }^{1}$, John Espino ${ }^{1 *}$. \\ ${ }^{l}$ Licenciatura en Ingeniería Electrónica y Telecomunicaciones, Centro Regional de Panamá Oeste \\ Universidad Tecnológica de Panamá
}

\begin{abstract}
Resumen La tecnología avanza exponencialmente, cambiando la manera en que las personas comprenden el mundo y las actividades que se realizan en él. Los teléfonos inteligentes se llevan gran parte de la atención de los estudiantes, afectando directamente su forma de aprendizaje. Aunque se estén llevando a cabo proyectos como la digitalización de libros, el uso de tableros digitales, entre otros, debemos pensar en la tecnología como el medio y no el fin, por lo tanto, no lograremos cambios notables en la educación si no cambiamos la manera de aprovechar e impartir las lecciones en los centros educativos del país. Este trabajo consiste en la creación de una aplicación móvil basada en resultados obtenidos de una investigación realizada en el área de la educación, específicamente en el uso y aprovechamiento de las tecnologías actuales para el aprendizaje, dentro de los centros educativos como fuera de estos. El proyecto llamado (TPEME) es una aplicación móvil la cual une la tecnología con la educación de una manera diferente, teniendo como fin mejorar el aprovechamiento y la calidad de las lecciones que se imparten actualmente en nuestros centros educativos.
\end{abstract}

Palabras clave Aprendizaje, crecimiento educacional, educación personalizada, aprovechar, tecnología.

\begin{abstract}
Technology advances exponentially, changing the way people understand the world and the activities that take place in it. Smartphones take much of the attention of students, directly affecting their way of learning. Although projects such as the digitization of books, the use of digital boards, among others, are being carried out, we must think of technology as the means and not the end, therefore, we will not achieve notable changes in education if we do not change the way to take advantage and teach the lessons in the country's educational centers. This work consists in the creation of a mobile application based on results obtained from an investigation carried out in the area of education, specifically in the use and use of current technologies for learning, within and outside educational centers. The project called (TPEME) is a mobile application that unites technology with education in a different way, aiming to improve the use and quality of the lessons currently taught in our educational centers.
\end{abstract}

Keywords Learning, educational growth, personalized education, free expression, Technology.

* Corresponding authors:jhon.espino@utp.ac.pa

\section{Introducción}

"Donde todos piensan igual nadie piensa mucho". La tecnología avanza a pasos agigantados, cada día vemos como estos avances cambian la manera como vivimos. Como ejemplos de estas nuevas tecnologías tenemos: celulares inteligentes, inteligencia artificial, redes sociales, internet [1].

Aunque existan tantos avances tecnológicos, la educación continua con un modelo de enseñanza antiguo, el cual tiene un avance de evolución muy lento casi nulo. Esto representa un problema debido a que los estudiantes no están siendo preparados para un mundo que está en constante desarrollo.

Esta razón nos impulsó a desarrollar un proyecto el cual llamamos "Tecnología para el mejoramiento educacional". TPEME, la cual es una aplicación que ayudará a mejorar exponencialmente la educación, está desarrollada en base a resultados de una investigación realizada por nosotros en la cual comprendimos aspectos importantes como tiempo dedicado a lecciones especificas por estudiantes, asignaturas difíciles de comprender, la manera en que se imparten las lecciones, el uso diario del celular y qué tanto aprenden los estudiantes con los métodos tradicionales de enseñanza.

\section{Antecedentes}

En 2008 se lanzó App Store por parte de la compañía de tecnología Apple, seguido de ella se lanzó Android market que pronto se renombro como Play Store. Estas son tiendas virtuales donde podemos adquirir aplicaciones para nuestros celulares inteligentes que también crean una fuente de ingresos a programadores que han desarrollado aplicaciones. A continuación, se presenta un resumen de bibliografía relevante. 
Más allá del autoinforme: herramientas para comparar el uso estimado y real del teléfono inteligente en el mundo real [2]

Los psicólogos suelen confiar en los datos de autoinforme al cuantificar el uso del teléfono móvil, a pesar de la poca evidencia de su validez. En este documento, exploramos la precisión del uso de estimaciones auto reportadas en comparación con el uso real de teléfonos inteligentes. También incluimos el código fuente para procesar y visualizar estos datos. Comparamos el uso real de 23 usuarios de teléfonos inteligentes en un período de dos semanas con estimaciones auto informadas y la escala de uso de problemas del teléfono móvil. Nuestros resultados indican que el tiempo estimado de uso de un teléfono inteligente puede ser una medida adecuada de uso, a menos que se requiera una mayor resolución de datos. Las estimaciones sobre el número de veces que un individuo usó su teléfono en un día típico no se correlacionaron con el uso real del teléfono inteligente. Ni la duración estimada ni el número de usos están correlacionados con la escala de uso de problemas del teléfono móvil.

\section{Resultados}

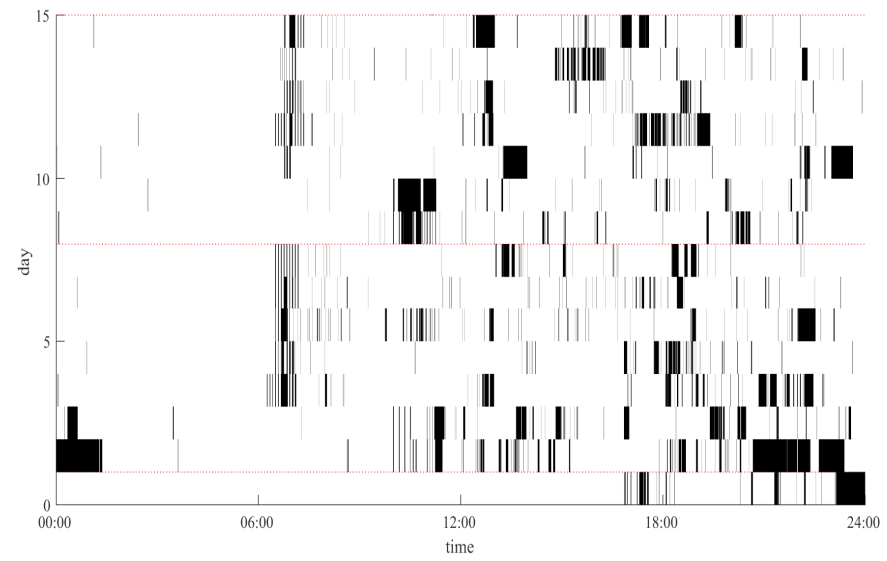

Figura 1 .Código de barras del uso del teléfono inteligente durante dos semanas.

Las áreas negras indican las horas en las que el teléfono estaba en uso y los sábados se indican con una línea discontinua roja. Las horas del despertador del día de la semana (y el dormitar) son claramente evidentes.

Datos objetivos

Se calculó el número medio diario de usos y la duración media de estas duraciones (incluida una mediana de la duración de todas las duraciones en un día) y una duración media diaria del uso del teléfono (duración diaria total) para cada participante. Los participantes utilizaron sus teléfonos una media de 84.68 veces por día $(\mathrm{SD}=55.23)$ y pasaron 5.05 horas cada día usando su teléfono inteligente ( $\mathrm{SD}=2.73)$. $\mathrm{La}$ duración del uso fue, como era de esperar, muy sesgada, con el $55 \%$ de todos los usos de menos de 30 segundos de duración ver figura 2.

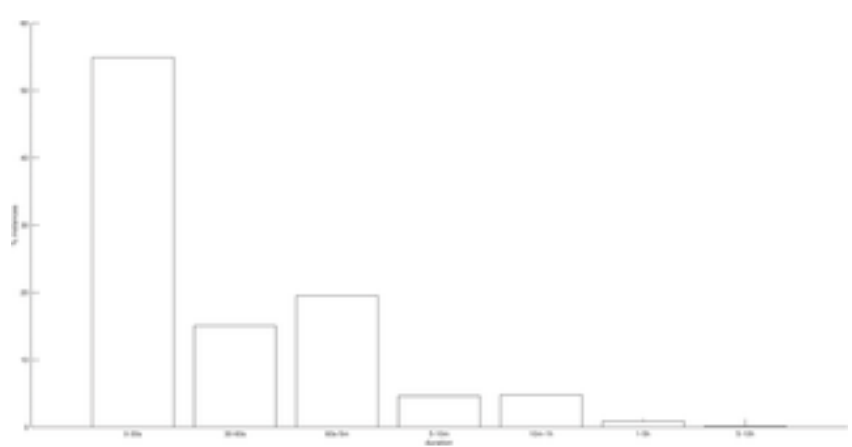

Figura 2. Porcentaje de usos categorizados por duración.

Esto ilustra la naturaleza altamente sesgada del uso de teléfonos inteligentes.

Los niveles estimados de uso de teléfonos inteligentes se han relacionado anteriormente con el sueño, las relaciones interpersonales, la seguridad al conducir y la personalidad. Aquí observamos que las estimaciones auto informadas del uso del teléfono se relacionan moderadamente con el comportamiento real en tales situaciones. Por el contrario, el número estimado de controles no mostró una relación clara con los usos reales; de hecho, los usos reales ascendieron a más del doble del número estimado. Es posible que nuestro tamaño de muestra limitado oscurezca un tamaño de efecto mayor. No obstante, sugerimos que el uso estimado puede no ser suficiente si se requiere una resolución de datos más alta, pero que las estimaciones del uso total probablemente sean adecuadas para muchos diseños de investigación. Sin embargo, para explorar los comportamientos de control, el número estimado de usos muestra poca confiabilidad para medir los usos reales

Uso de la tecnología celular por el alumnado y su influencia en el aprendizaje activo dentro del salón de clases [3]

La investigación trata sobre el uso de la tecnología celular por el alumnado y su influencia en el aprendizaje activo dentro del salón de clases, lo cual es de suma importancia conocer ya que se pueden presentar varias dificultades en el aprendizaje de los alumnos/as a causa del uso que se le da al teléfono celular.

\section{Objetivo}

Indagar la influencia del uso de la tecnología celular por el alumnado en el aprendizaje activo dentro del salón de clases del tercer ciclo.

Identificar si la dimensión instrumental del teléfono celular incide en la participación del alumnado del tercer ciclo de los centros educativos participantes.

Conocer la influencia del celular en su dimensión formativa de identidad del alumno/a en sus relaciones interpersonales dentro del salón de clases.

Analizar el uso cotidiano del celular y su influencia en el desempeño de las actividades educativas dentro del salón de 
clase por el alumnado del tercer ciclo de los centros educativos participantes.

¿El uso de la tecnología celular influye en el aprendizaje activo del alumno/a dentro del salón de clases?

La presente investigación fue de tipo correlacional, ya que este estudio tuvo como propósito el de conocer la relación que existe entre dos variables, la dependiente y la independiente. En este caso la variable independiente es: uso de la tecnología celular y la variable dependiente es el aprendizaje activo del alumno dentro del salón de clases.

\section{Resultados}

En cuanto al ingreso de cuenta de usuario de redes sociales del celular, los alumnos/as expresaron en un 54\% que, si ingresan desde su teléfono móvil para revisar su Facebook, Twitter y chat, mientras que un $46 \%$ no ingresan desde un teléfono celular a una cuenta de redes sociales. Por otra parte, se pudo constatar que las horas predilectas de los jóvenes en el ingreso a las redes sociales son de 8:00 a. m. a 11:00 a. m. en la mañana; de 3:00 p. m. a 5:00 p. m. en la tarde y de 6:00 p. m. a 11:00 p. m. en la noche, por lo cual se puede reflexionar que un joven pasa un promedio de alrededor de cuatro horas en su teléfono celular actualizando sus redes sociales

Según los docentes entrevistados, el ingreso que hace el alumno con el teléfono celular en el salón de clases provoca una interferencia, ya que interrumpe e irrespeta al docente y a sus demás compañeros, generando una desconcentración e irresponsabilidad a la hora de realizar las tareas asignadas trayéndole como consecuencia un bajo promedio y por ende un bajo rendimiento académico. En cuanto al envío y recepción de llamadas o mensajes de texto mencionaron que es evidente que el alumno/a se encuentra más enfocado en atenderlos que en prestar atención en las clases o en trabajar en las actividades que se realizan en el aula, como consecuencia a esto el alumno/a se vuelve lento a la hora de resolver las actividades que le asigna el docente o si en algunos casos las logra completar estas están deficientes y tienen una baja calidad debido a que el alumno/a no pone el esfuerzo suficiente y presenta ansiedad de terminar las actividades con rapidez aunque no estén bien realizadas.

\section{Conclusiones}

Se sugiere que los docentes utilicen estrategias de $M$ Learning como: softwares convertidores de formatos de video y audio compatibles a los teléfonos celulares, uso de portales educativos, creación de podcast, uso del sistema "Wapeduc", así como la creación de weblogs propios de los profesores y las materias que imparten, y el aprovechamiento de plataformas como Java Me y Androide en teléfonos celulares para la aplicación de programas educativos.

\section{Metodología}

\section{1- Observación:}

El desarrollo tecnológico cada día crece más, dando una forma diferente de comprender los desafíos que se presentan a lo largo de la vida. Podemos decir que la mejor manera de prepararnos para el futuro es mediante la educación, sin embargo, la tecnología se convierte en un factor considerable en contra si no se aprovecha de la manera correcta, afecta la concentración y la motivación de los estudiantes dentro y fuera los salones de clases.

Sabemos que la mayor parte de la población tienen celulares inteligentes que podemos aprovechar creando una aplicación para mejorar el sistema de preparación dentro y fuera de las aulas de clases en nuestro país para generar un impacto positivo en el futuro.

\section{2- Pregunta:}

¿Cómo debemos diseñar una aplicación inteligente que pueda ayudar a los estudiantes a comprender, aprovechar, mejorar las clases y mantener ese interés por el aprendizaje dentro y fuera de las aulas de nuestro país?

\section{3- Hipótesis:}

El avance de la educación se logra cambiando la forma como se imparten las clases, por una más personalizada, interactiva y de calidad utilizando la tecnología como herramienta.

Aprovechando las características y sensores que poseen los celulares inteligentes y tabletas generamos un software capaz de adaptarse a cada estudiante mejorando la forma en que desarrolla sus capacidades y aprendizaje.

\section{Construcción de la aplicación \\ 1- Inicios}

Inicialmente el entorno de programación utilizado para (TPEME) fue App Inventor figura 3, el cual es un entorno desarrollado por el (MIT) basado en bloques. Al utilizar este entorno se nos facilitó la creación de la interfaz con la cual llevamos a cabo la primera prueba realizada con los dos niños de cinco y seis años respectivamente.

Lo que diferencia nuestro software es la manera cómo interpreta la capacidad de aprendizaje del estudiante proporcionándole automáticamente un proceso acorde a lo obtenido. 


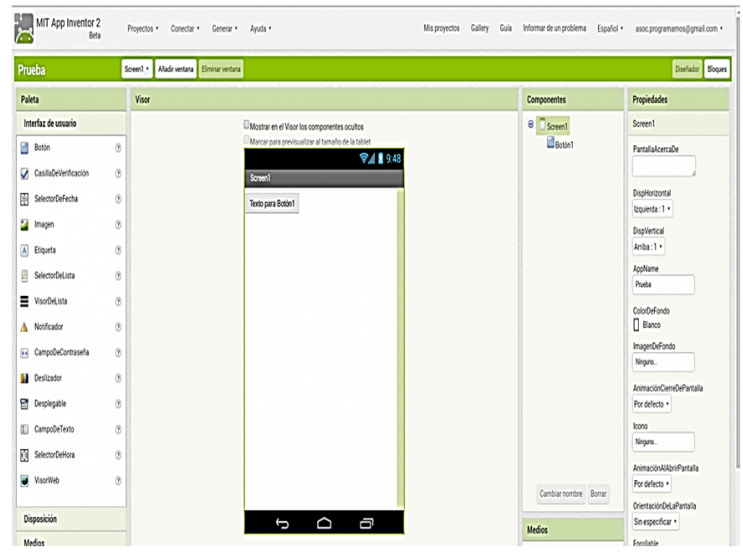

Figura 3. Entorno de desarrollo App Inventor.

\section{2- Funcionamiento}

a- Al iniciar el software debes crear una cuenta de usuario, aquí se debe seleccionar si es estudiante u orientador utilizando tus datos personales, los cuales se guardan en una base de datos privada. Estos datos sirven para proteger tu sesión y brindar conocimiento de la persona que utiliza la aplicación a su respectivo consejero.

b- En el siguiente paso debes realizar una prueba psicológica, la cual se realiza con el fin de conocer generalmente las preferencias, hábitos, fortalezas y debilidades de los estudiantes para optimizar la aplicación en base a su usuario.

c- El siguiente paso es en el menú de cursos, aquí puede escoger uno de los cursos que se encuentran disponibles, una vez seleccionado el curso tendrá que realizar una prueba, la cual tiene el objetivo de conocer el nivel de comprensión que inicialmente tiene el estudiante y posicionarlo en un nivel dentro del curso. Si el nivel inicial es superior a los niveles del curso, automáticamente se le recomendará un curso más avanzado.

\section{Funcionamiento de los cursos dentro de la aplicación}

Dentro del curso, el software funciona con videos, los cuales se le presentan al estudiante cuando pasa de nivel de la siguiente manera:

* El curso cuenta con tres profesores diferentes los cuales han hecho videos para el curso del mismo tema, pero explicando de diferentes formas.

* El primer video del curso lo presenta la aplicación aleatoriamente.

* Al finalizar el video se presenta una prueba sobre el tema, la cual se debe aprobar con más del $69 \%$ de respuestas correctas.
* Si el estudiante aprueba, se le suman puntos a su contador y la aplicación lo pasa a otro video del siguiente tema, pero con el mismo profesor.

- Esto nos dice que el estudiante entendió correctamente el método de enseñanza del profesor y puede seguir con este.

* Si el estudiante no supera la prueba el software no suma ni resta puntos y automáticamente lo cambia de profesor, pero conservando el tema actual,

- Esto para que el tema sea aprendido con una forma diferente de enseñanza.

* El software durante todo el curso recoge datos como tiempo en responder preguntas, veces que repite el video, cambio de profesor, entre otros y los convierte en datos que se envía al consejero, el cual puede tomar acciones al respecto.

* Este ciclo se repite creando un ambiente fluido dentro de la aplicación.

* Si el estudiante sale del curso, al entrar se repite el proceso de ubicación en niveles para reforzar sus conocimientos.

* La aplicación funciona con internet, por lo tanto, se puede tomar cursos tanto fuera del aula de clase como dentro, y seguirá llegando los datos al orientador respectivo.

* De igual forma se puede salir de un curso e iniciar otro.

\section{Trascripción a Código en Android Studio}

Decidimos escribir la aplicación en código por dos motivos:

1- Más control de los procesos internos de la aplicación.

2- Agregar funciones necesarias para el correcto funcionamiento de la aplicación.

Utilizamos el SDK Android studio (figura 4), por ser la mejor opción para el desarrollo de aplicaciones en Android.

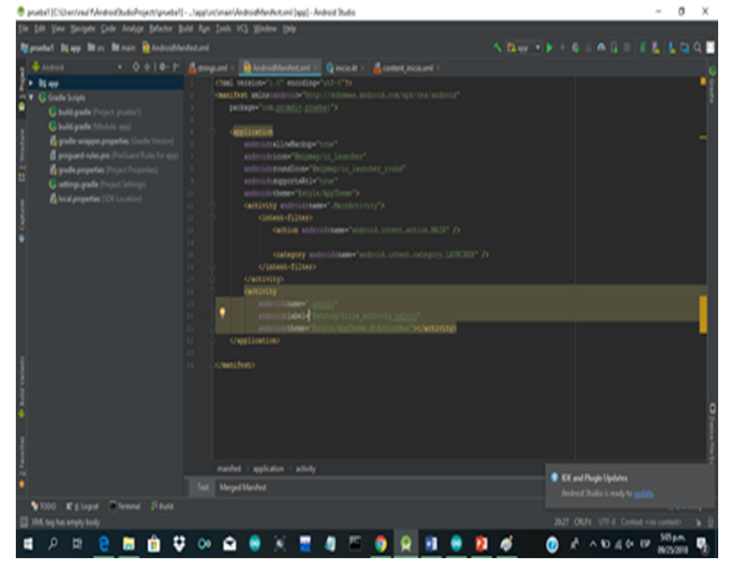

Figura 4. Entorno de desarrollo Android Studio. 
Este proceso no cambió la manera de funcionar de la aplicación, mejoró alguno de los procesos optimizándolos.

\section{Experimentación}

Realizamos dos experimentos, en los cuales comparamos la funcionalidad de la aplicación frente al método tradicional de enseñanza.

\section{Experimento 1}

Se escogen dos niños, con el permiso de sus padres, el primero tiene seis años y está en primer grado, la siguiente es una niña de cinco años que está en kínder. A los dos niños se enseña a multiplicar números menores a 20, con métodos diferentes.

Nota: Los dos niños ya saben sumar.

1- Primer método:

Materiales: Lápiz, cuaderno, tablas de multiplicar, borrador, tablero, marcadores y sacapuntas.

Método de repetición que normalmente utilizan para enseñar las tablas de multiplicar, consiste en repetir el número a multiplicar "por" el número multiplicador "igual" número resultante de la operación. El método es aplicado por uno de los investigadores y uno de los padres del niño.

2- Segundo método:

Materiales: Tablet (Android) con (TPEME) instalado, Smart phone (Android).

Método basado en el nivel de aprendizaje del niño con ayuda de las (TPEME), el nivel de aprendizaje es medido por una prueba dentro de la App en una Tablet, este test fue diseñado exclusivamente para niños de estas edades (cinco años a siete años), consiste en imágenes y preguntas supervisadas por orientadores, en este caso uno de los investigadores y uno de los padres de la niña.

- Luego de obtener los datos de la niña, desde test de la app de (TPEME) imagen (1) se envían al Smart phone del orientador automáticamente.

Luego se genera un juego que enseña la multiplicación mediante problemas que evolucionan según el ritmo al cual niño evoluciona, esto de una forma divertida y personalizada.

$$
\text { Respuesta }=6
$$

Además, en todo memento el orientador está dándole seguimiento al progreso analizando los datos y respondiendo preguntas realizadas por el niño.

\section{Resultados de experimento 1}

Nota: Los resultados en esta experimentación fueron tomados en un tiempo de dos horas. Además, estos resultados se han obtenido con el uso de los métodos son solo al principio de la investigación, en los días próximos se obtendrán más datos de estudiantes, para obtener resultados amplios.

Los datos son evaluados por los padres de los niños y por los investigadores basándose en una prueba que consiste en preguntas orales y problemas escritos sobre multiplicación utilizando objetos conocidos por los niños como frutas, balones deportivos, etc.

También se midió el tiempo en que respondían (los orientadores no permitieron que existiera algún tipo de presión hacia los niños).

Las preguntas de la prueba fueron las siguientes:

¿Si Saúl compra dos paquetes de galletas Pascual de chocolate cuantas galletas lleva?

Respuesta $=8$.

¿Cuánto es una docena de huevos?

Respuesta $=12$.

¿Cuánto son 3 monedas de 5 centavos?

Respuesta $=15$ centavos.

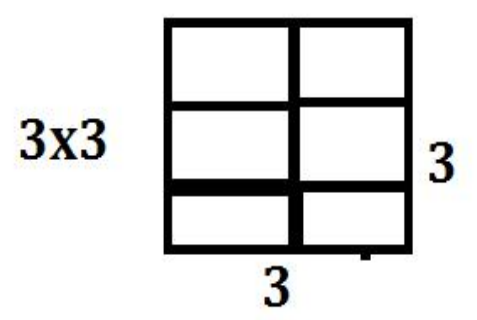

Respuesta $=9$.
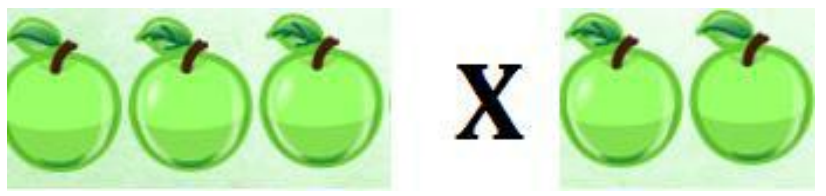
A. Para el primer método lencio (et al): Tecnología para el mejoramiento educacional (TPEME)

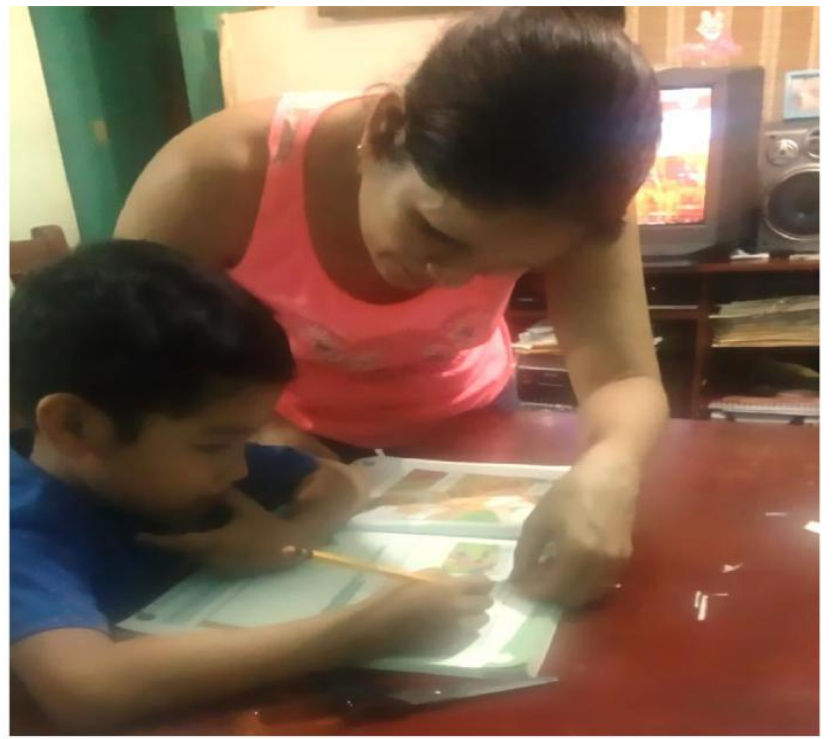

Figura 5. Estudiante uno.

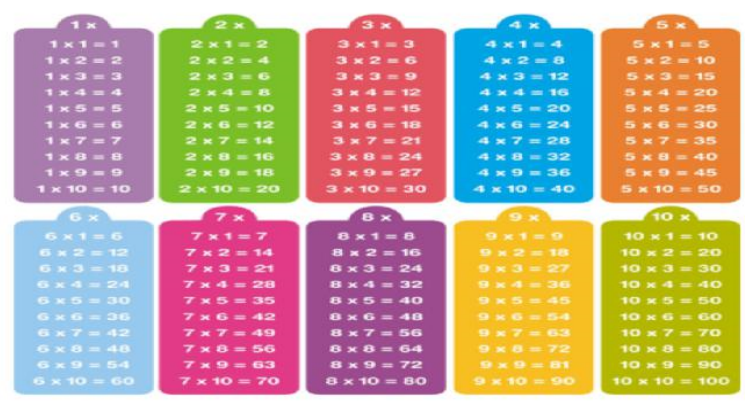

Figura 6. Tablas de multiplicar.

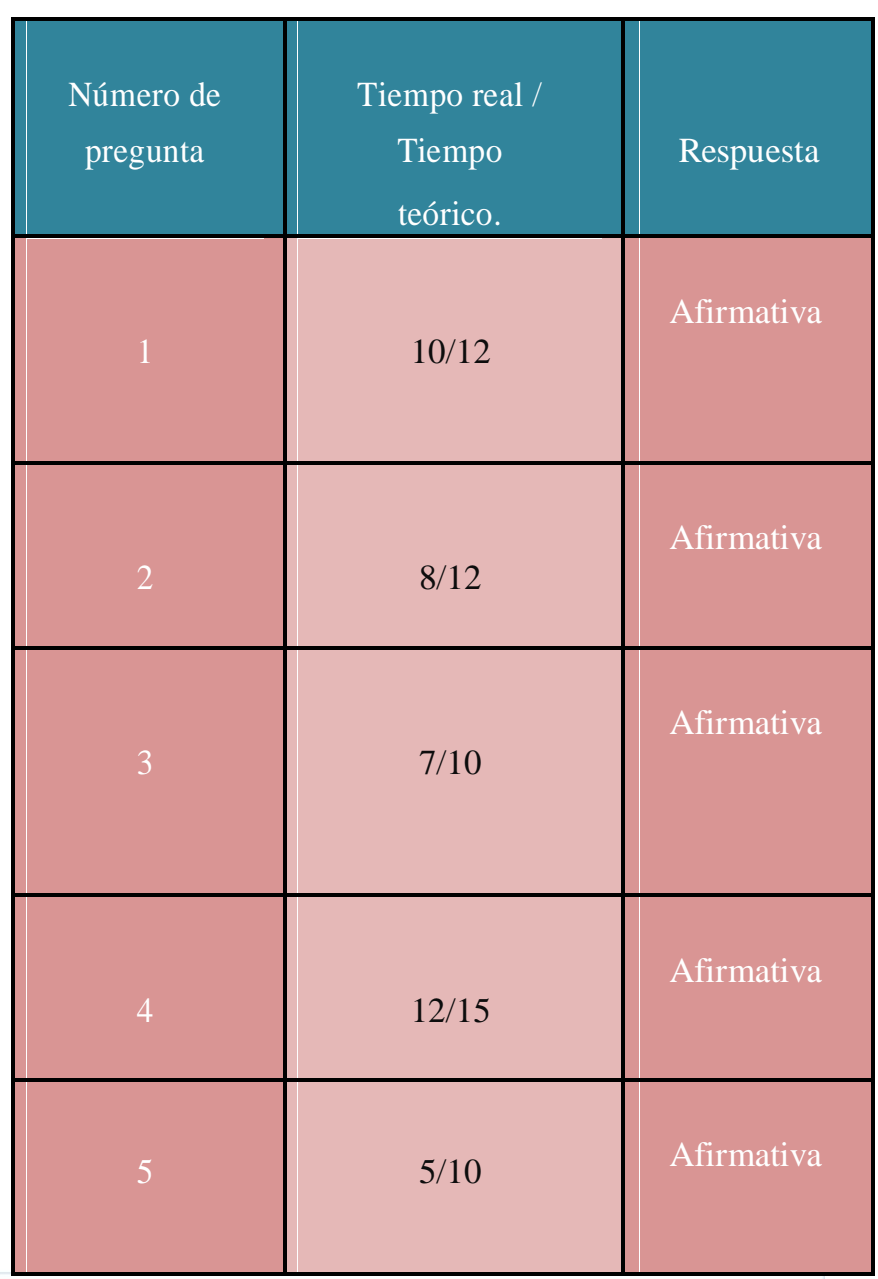

Tiempo teórico

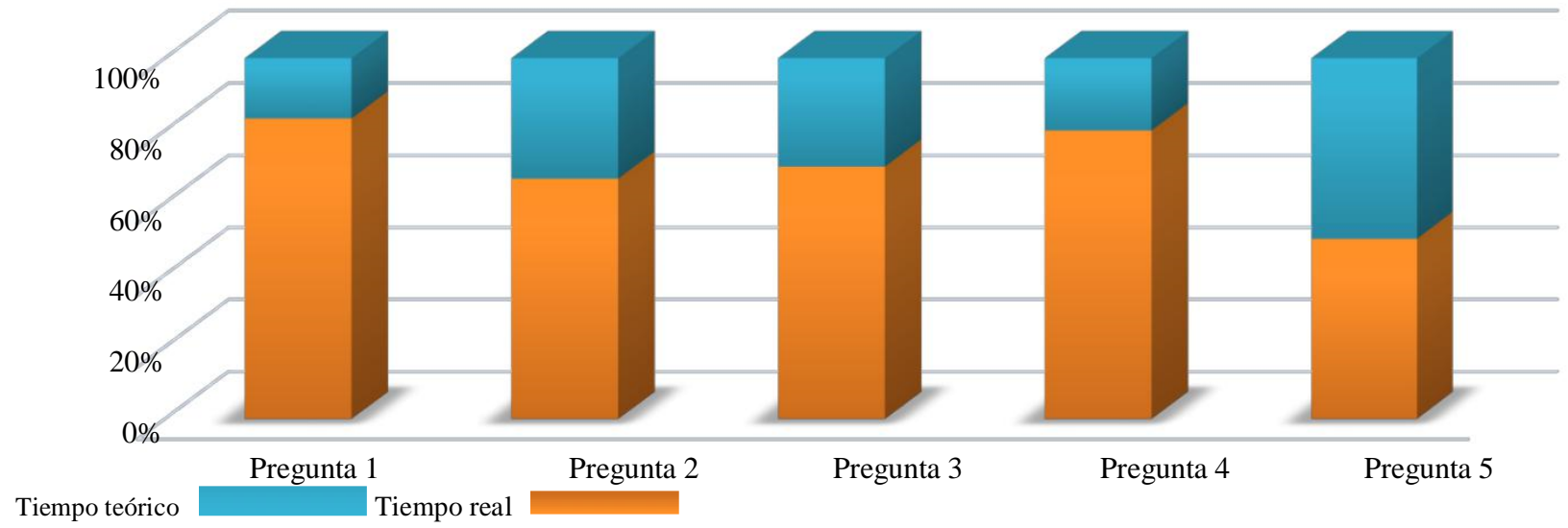

Gráfico 1. Uso del tiempo real/Teórico. 


\section{B. Para el segundo método los resultados fueron:}

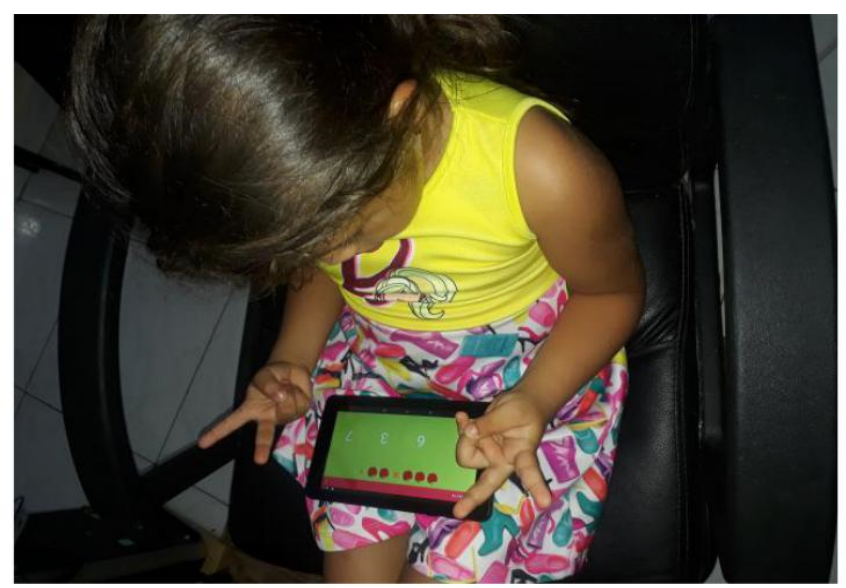

Figura 7. Estudiante dos.

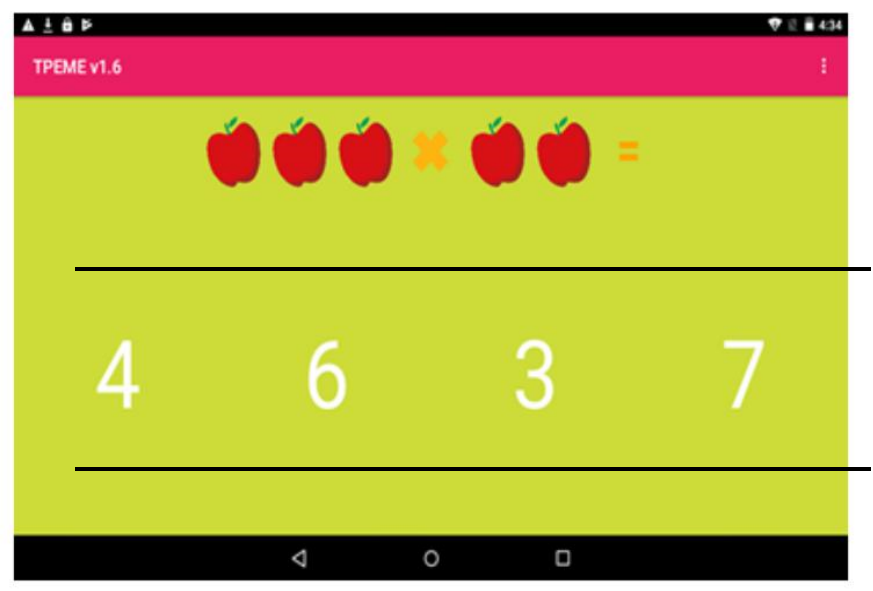

Figura 8. Interfaz de TPEME

\begin{tabular}{|c|c|c|}
\hline $\begin{array}{l}\text { Número de } \\
\text { pregunta }\end{array}$ & $\begin{array}{c}\text { Tiempo real / } \\
\text { Tiempo } \\
\text { teórico. }\end{array}$ & Respuesta \\
\hline 1 & $6 / 12$ & Afirmativa \\
\hline 2 & $3 / 12$ & Afirmativa \\
\hline 3 & $4 / 10$ & Afirmativa \\
\hline 4 & $9 / 15$ & Afirmativa \\
\hline 5 & $3 / 10$ & Afirmativa \\
\hline
\end{tabular}

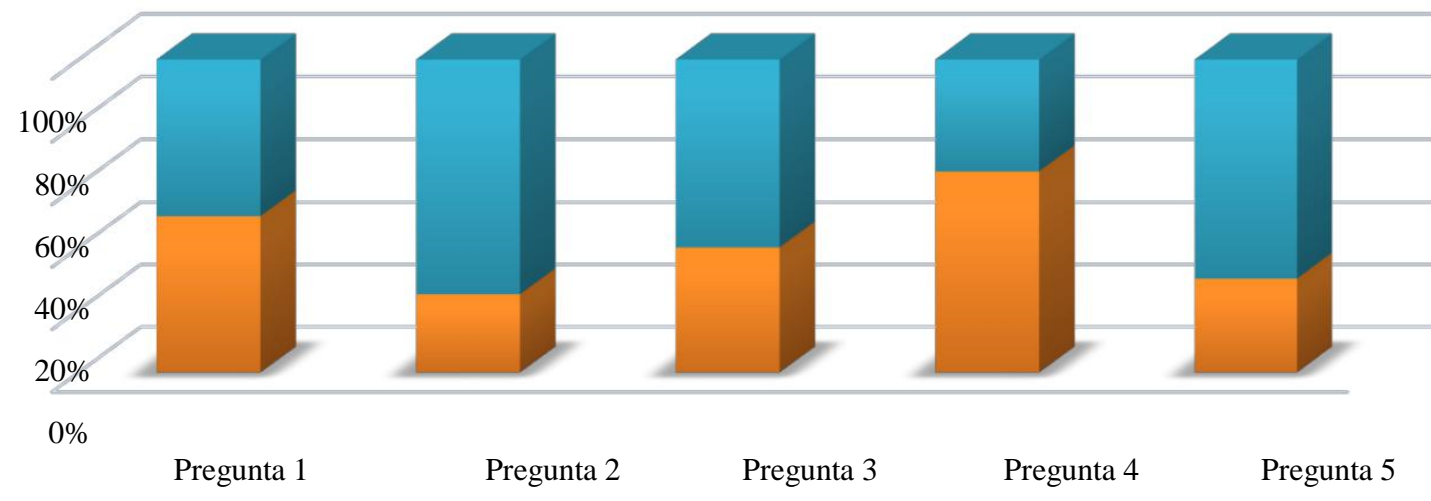

Gráfico 2. Uso del tiempo real/Teórico

Tiempo real $\quad$ Tiempo teórico 


\section{Experimento dos}

La prueba de rendimiento de la aplicación de TPEME fue realizada en el C. E. B. Victoria D'Spinay.

Este experimento lo llevamos a cabo con más estudiantes de niveles entre tres y cinco grado.

El experimento se basó en pruebas de matemáticas para las cuales formamos grupos de estudiantes de diferentes niveles y los llamamos grupo A y B.

El grupo A figura 9, obtuvo explicación por uno de los investigadores el cual les mostró como resolver problemas matemáticos de división, suma, resta, multiplicación y problemas combinados.

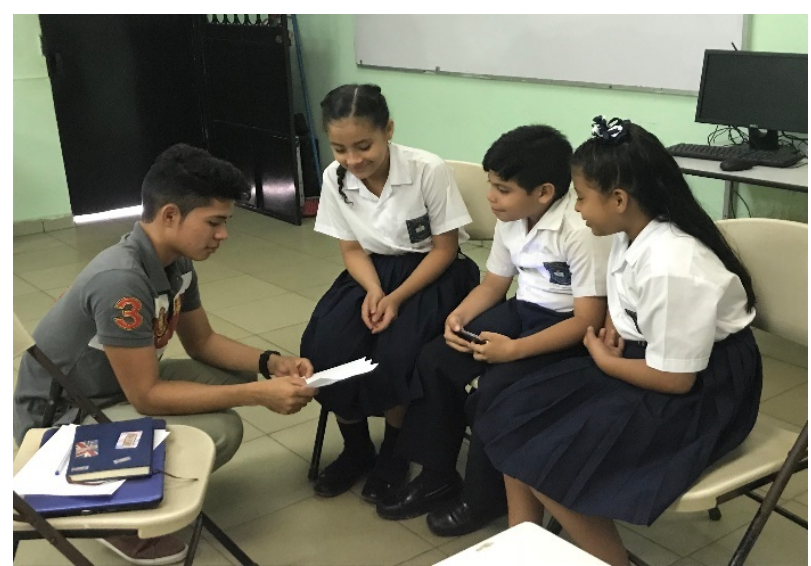

Figura 9. Aquí se puede ver cuando los estudiantes estaban haciendo las pruebas debidamente con el primer método.

El grupo B figura 10 obtuvo explicación por medio de la aplicación (TPEME) de cómo resolver problemas matemáticos de división, suma, resta, multiplicación y problemas combinados.

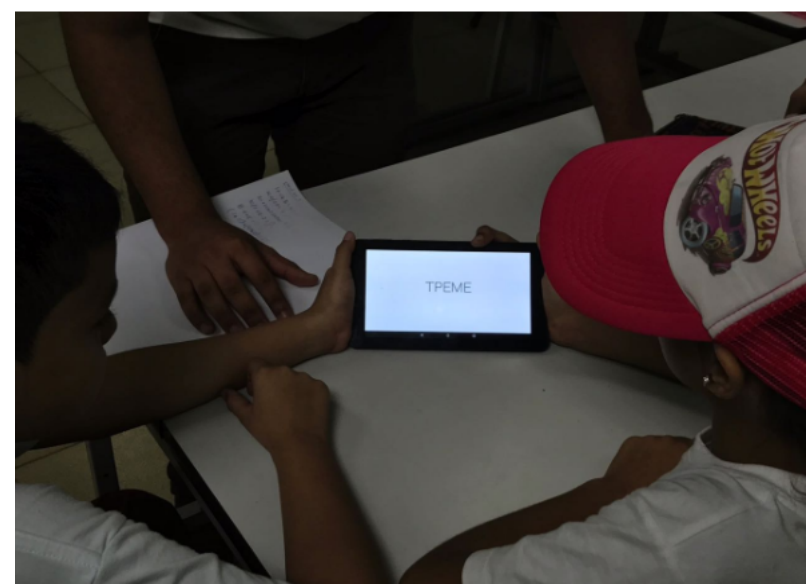

Figura 10. Método (TPEME) con ayuda de una tableta.

\section{Prueba de experimento}

Luego de un tiempo los grupos de estudiantes se reunieron y se les dio una hoja con los mismos problemas a todos y se les pidió que los resolvieran y si no sabían alguno podían dejarlo sin resolver.

\section{Resultados de experimento dos}

Para esta prueba se usó la aplicación de TPEME orientado a matemáticas. La prueba se aplicó a:

tres estudiantes de $3^{\circ}$ grado.

tres estudiantes de $5^{\circ}$ grado.

Estos datos se tomaron en base al tiempo de respuesta y respuesta correcta de los estudiantes.

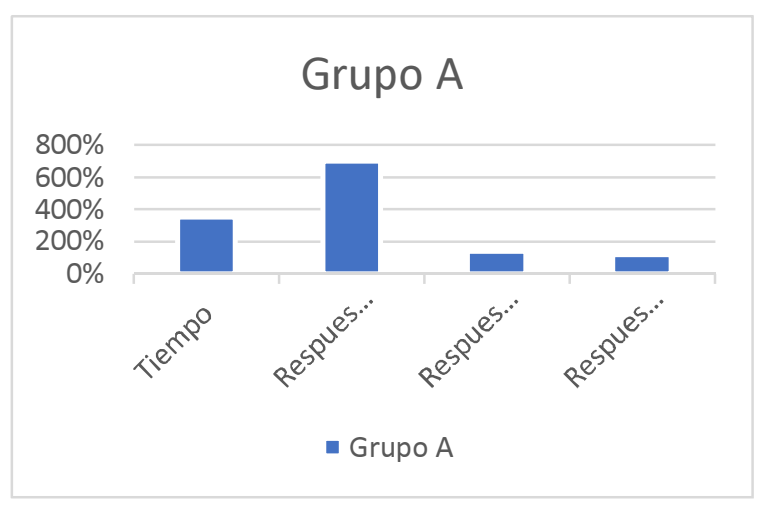

Gráfico 3. Grupo A.

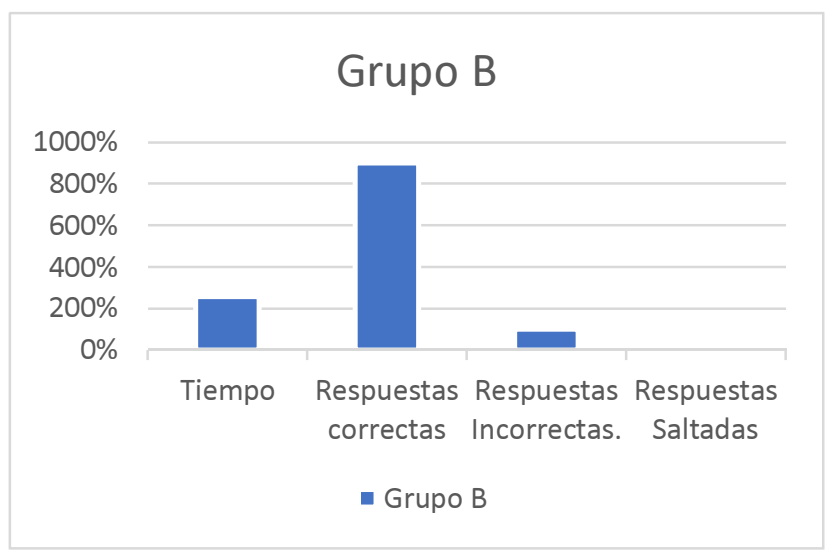

Gráfico 3. Grupo B.

\section{Experimento 3}

Este experimento se realizó en la feria de matemáticas en el colegio San Gabriel de la Dolorosa.

Para este experimento modificamos la interfaz de la aplicación y colocamos un gato azul de logo, con el fin de llamar la atención de los presentes en la actividad.

En esta actividad disponíamos de un stand en la feria por la cual los estudiantes y profesores pasaban recorriendo información, los interesados al pasar les llamaba la atención el diseño de la aplicación y lo que estábamos explicando, al inicio solo pedíamos que utilizaran la aplicación y nos respondieran tres sencillas preguntas en una escala de cero a cien.

Las preguntas formuladas fueron: 
¿De uno a cien qué tan agradable es?

¿De uno a cien qué tanto crees que entendiste del tema explicado?

¿De uno a cien qué puntuaciones le darías a la idea en general?

Se les hicieron estas preguntas a 31 personas entre estudiantes.

Figura 12. De diversos niveles y profesores (figura 11), en diferentes áreas como física, matemática, entre otros, de los cuales obtuvimos los siguientes resultados.

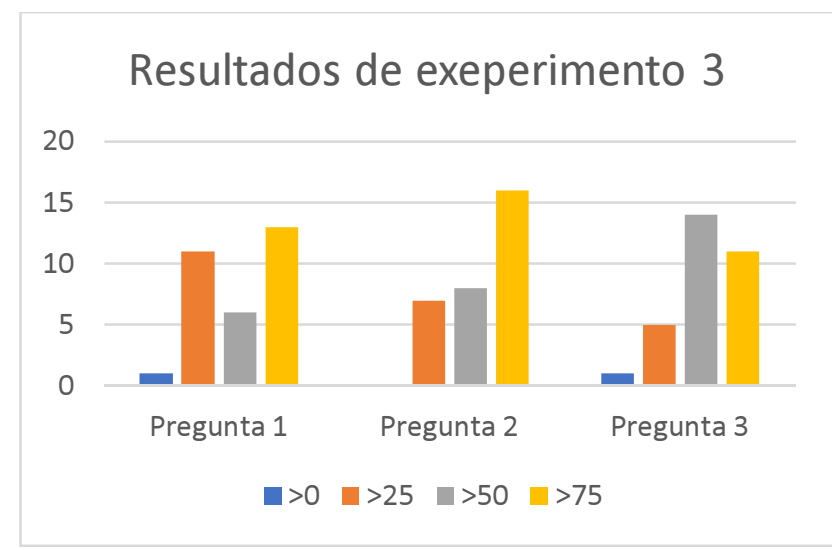

Gráfico 4. respuesta de 31 personas entrevistadas.

Estos resultados nos confirman el interés y la aceptación por parte de profesores y estudiantes con respecto a la idea de enseñanza de la aplicación. figura 13.

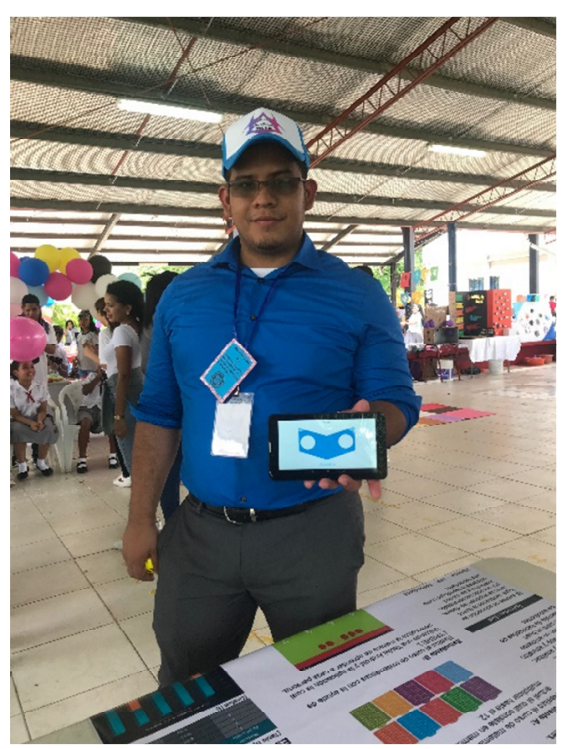

Figura 11. Profesor de Física apoya la idea.

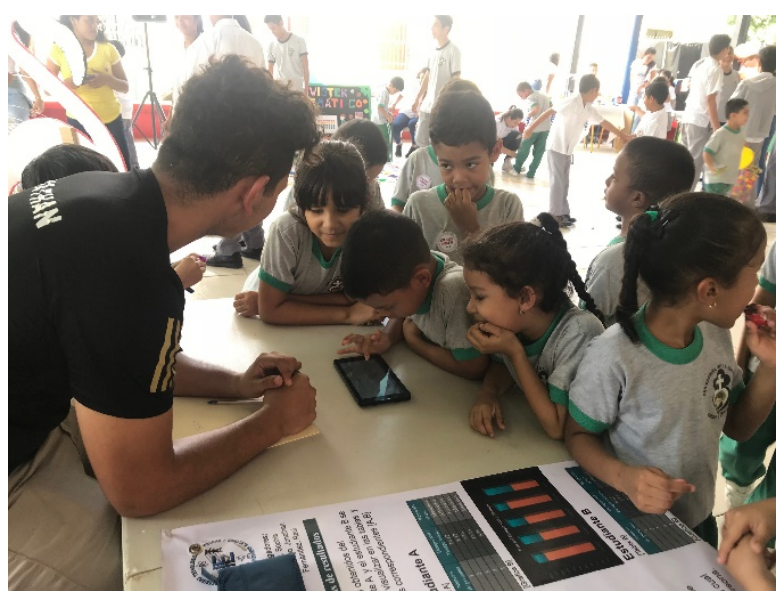

Figura 12. Estudiantes motivados con la aplicación TPEME.

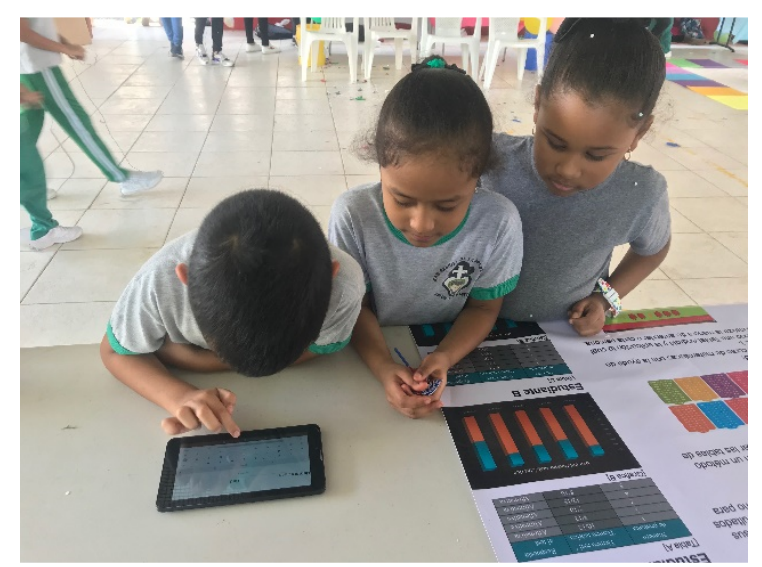

Figura 13. Estudiantes utilizando la aplicación de TPEME.

\section{Conclusiones}

"El sistema educativo actual es el resultado de varios años de transición progresiva y exponencial. En la actualidad es necesario un cambio en las metodologías y en los contenidos impartidos para poder afrontar con garantías el complejo y diverso futuro que nos aguarda [4]".

El dato recogido en los tres experimentos nos refleja claramente que una aplicación tiene potencial en el ámbito de la educación, siempre y cuando cumpla con un método de enseñanza innovador y amigable a los estudiantes.

Aunque se deben realizar más estudios con diferentes estudiantes, podemos decir que la hipótesis es confirmada. Por lo tanto, al cambiar el estilo de enseñanza e implementando un verdadero uso de la tecnología como herramienta a la hora de enseñar, podemos hacer que los estudiantes disfruten la educación de manera que esto cree un ambiente de motivación que resultara en un cambio radical en el futuro, mejorando así la calidad de vida de todos. 
También podemos recalcar que la tecnología no es el fin sino el medio, un medio que debemos aprovechar si queremos mejorar el futuro de los estudiantes en nuestro país.

\section{REFERENCIAS}

[1] j. C. RUIZ, «eldia.es,» 16 Julio 2017. [En línea]. Available: http://eldia.es/criterios/2017-07-16/12-Donde-todos-

[2] D. A. E. H. S. L. P. Sally Andrews, «Más allá del autoinforme: herramientas para comparar el uso estimado y real del teléfono inteligente en el mundo real,» 28 Octubre 2015. [En línea]. Available:

https://journals.plos.org/plosone/article?id=10.1371/journal.p one.0139004.

[3] D. L. S. Garcia, S. A. V. d. León y I. E. V. Gamez, «Uso de la tecnología celular por el alumnado y su influencia en el aprendizaje activo dentro del salón de clases.,», 2012. [En línea]. Available: http://ri.ues.edu.sv/2275. [Último acceso: 25 9 2018].

[4] N. Rivera, «hipertextual.com,» 13 octubre 2016. [En línea]. Available: https://hipertextual.com/especiales/tecnologia-eneducacion. [Último acceso: 14 junio 2018].

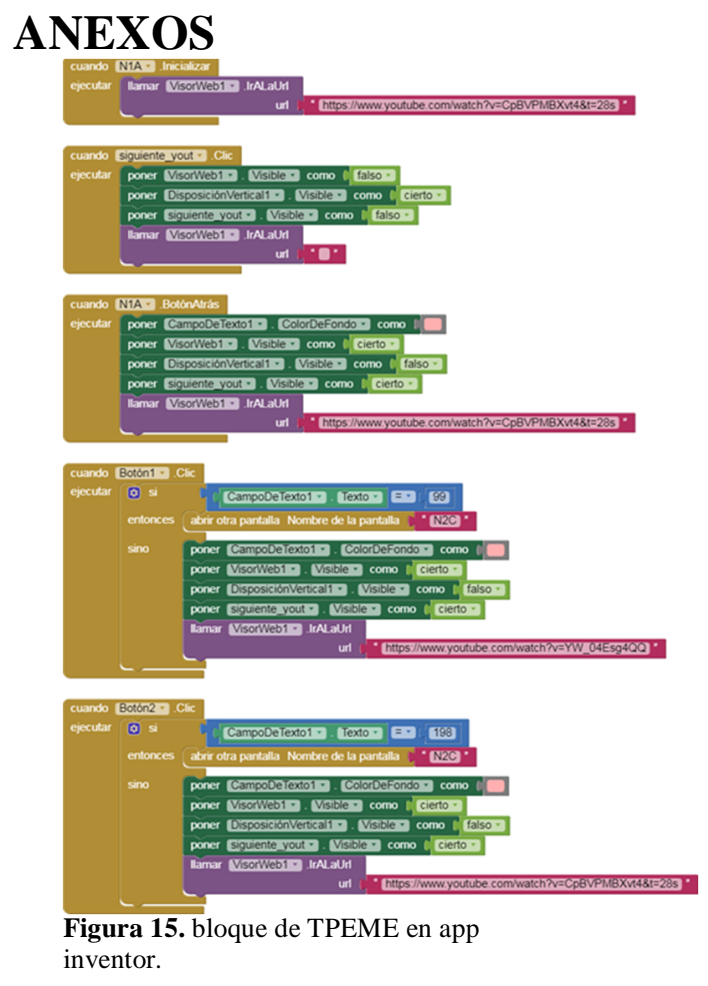

